\title{
A vivência da mulher no período do climatério: revisão integrativa
}

\author{
The experience of women in the climate period: integrative review \\ La experiencia de las mujeres en el período climático: revisión integrativa
}

Recebido: 26/09/2021 | Revisado: 02/10/2021 | Aceito: 05/10/2021 | Publicado: 10/10/2021

\author{
Alanna Nascimento Alvarenga \\ ORCID: https://orcid.org/0000-0003-2286-1349 \\ Centro Universitário Santo Agostinho, Brasil \\ E-mail: alannaalvarenga5@gmail.com \\ Cinara Lima Visgueira \\ ORCID: https://orcid.org/0000-0003-4675-269X \\ Centro Universitário Santo Agostinho, Brasil \\ E-mail: cinaralvisgueira@gmail.com \\ Raquel Vilanova Araújo \\ ORCID: https://orcid.org/0000-0002-1752-296X \\ Centro Universitário Santo Agostinho, Brasil \\ E-mail: raquelvilanova@unifsa.com.br
}

\begin{abstract}
Resumo
O climatério se caracteriza de acordo como um estágio de mudança entre a fase reprodutiva e não reprodutiva do ciclo de vida da mulher, de forma não patológica. O objetivo deste estudo é compreender como as mulheres vivenciam o climatério e conhecer as repercussões do climatério em sua vida. Trata-se de uma revisão integrativa da literatura realizada com base na estratégia PICo, realizada na base de dados da Lilacs - Literatura Latino Americana e do Caribe em Ciência em Saúde e Bdenf - Base de Dados em Enfermagem com os descritores Mesh, mulher, vivência, climatério, mudança de vida. Foram resgatados 77 estudos após critérios de inclusão e exclusão, obteve-se 25 artigos que compuseram a amostra final. Foram construídas três categorias: Vivenciando os sinais e sintomas do climatério; As repercussões do climatério na vida social da mulher e as repercussões do climatério nos aspectos psicológicos da mulher. Foi evidenciado que cada mulher experencia o climatério de maneira singular, assim como as sintomatologias que têm um impacto diferente na vida de cada uma.
\end{abstract}

Palavras-chave: Climatério; Mulher; Vivência.

\begin{abstract}
Climacteric is characterized according to a stage of change between the reproductive and non-reproductive phases of the woman's life cycle, in a non-pathological way. Objective: to understand how women experience the climacteric and to know the repercussions of the climacteric in their lives. This is an integrative literature review based on the PICo strategy, carried out in the database of Lilacs - Latin American and Caribbean Literature in Health Science and Bdenf - Database in Nursing with the descriptors Mesh, woman, experience, climacteric, change of life. 77 studies were retrieved after inclusion and exclusion criteria, obtaining 25 articles that made up the final sample. Three categories were constructed: Experiencing the signs and symptoms of menopause; The repercussions of menopause in women's social life and the repercussions of menopause on women's psychological aspects. It was shown that each woman experiences menopause in a unique way, as well as the symptoms that have a different impact on each woman's life.
\end{abstract}

Keywords: Climacteric; Woman; Experience.

\section{Resumen}

El climaterio se caracteriza por una etapa de cambio entre las fases reproductiva y no reproductiva del ciclo vital de la mujer, de forma no patológica. Comprender cómo las mujeres experimentan el climaterio y conocer las repercusiones del climaterio en sus vidas. Se trata de una revisión integradora de la literatura basada en la estrategia PICo, realizada en la base de datos Lilacs - Literatura Latinoamericana y Caribeña en Ciencias de la Salud y Bdenf - Base de Datos en Enfermería con los descriptores Malla, mujer, experiencia, climaterio, cambio de vida. Se recuperaron 77 estudios después de los criterios de inclusión y exclusión, obteniendo 25 artículos que conformaron la muestra final. Se construyeron tres categorías: Experimentar los signos y síntomas de la menopausia; Las repercusiones de la menopausia en la vida social de las mujeres y las repercusiones de la menopausia en los aspectos psicológicos de las mujeres. Se demostró que cada mujer vive la menopausia de una manera única, así como los síntomas que repercuten de manera diferente en la vida de cada mujer.

Palabras clave: Climatérico; Mujer; Experiencia. 


\section{Introdução}

O climatério se caracteriza como um estágio de mudança entre a fase reprodutiva e não reprodutiva do ciclo de vida da mulher, de forma não patológica. Portela, Silva, Rodrigues, Oliveira e Filho (2017) afirma que com o aumento da expectativa de vida um terço do ciclo vital de uma mulher passará no climatério. Assim, Assunção, Íris, Barreto, Gonçalves e Dias (2017) diz que o período do climatério é evidenciado por alterações hormonais que, logo acarretarão em implicações em diversos aspectos na vida da mulher, como biológicas, psicológicas, sociais e culturais.

O diagnóstico da menopausa é feito de forma pregressa (depois de 12 meses de amenorreia). A menopausa é dividida em dois períodos, a pré-menopausa, período anterior a última menstruação, e pós-menopausa, tempo de 12 a 24 meses posterior a última menstruação, caracterizando a perimenopausa (Maciel et al., 2021). O momento de alteração do período reprodutivo para o não reprodutivo inicia-se por volta dos 40 anos de idade e se prolonga até os 55 anos, e que cerca de 20 a $25 \%$ das mulheres no climatério não apresentam sintomas, e reforça que os sintomas, quando surgem pode ser consequência da insuficiência ovariana (Vieira et al., 2018).

A fase do climatério envolve uma série de modificações biopsicossociais, que podem ser vividas e enfrentadas com medo e ansiedade por algumas mulheres (Meira, Morais, Sousa \& Ferreira, 2020). Este período inclui, dentre as elas como, a beleza física típica da juventude, a perda do potencial reprodutivo, o envelhecimento aparente, além de outras crenças socioculturais negativas outorgada à mulher nessa fase (Silva et al., 2016). Ademias encontram-se alterações relacionadas ao estilo de vida adotado, à queda ou ao desequilíbrio hormonal, ao estado geral da mulher, à autopercepção corporal que podem interferir o modo de vivenciar essa fase (Silva et al., 2016).

Quando o climatério ou menopausa, ocorre de forma precoce, são mais intensas as alterações no humor, irritabilidade e nervosismo (Oliveira et al., 2019), e relacionadas ao desenvolvimento de doenças cardiovasculares e osteoporose, em virtude da insuficiência hormonal (Maciel, 2021). De acordo com Souza et al., (2017) e Silva, Freire e Nascimento (2019), o período do climatério pode ser vivenciado de forma patológica por mulheres que apresentam perda na qualidade de vida e ver este como momento como um período de crise, dúvidas e medos, insegurança, relacionada ao envelhecimento, fim da vida reprodutiva e sexualidade.

A importância de se estudar acerca do climatério e seus impactos, de acordo com Carvalho, Júnior, Parente e Sales, (2018), se torna essencial pois esse período conforme os sintomas aparecem, podem alterar a qualidade das relações estabelecidas da mulher no meio profissional, familiar, influenciando o estado satisfatório da mulher afetando sua rotina habitual e/ou relações interpessoais.

É essencial também citar os aspectos psicológicas dessas mulheres, pois o fato de estarem no climatério principalmente na pós-menopausa, pode ocorrer um período da diminuição da produtividade, feminilidade e de grande desconforto (Xavier \& Trindade, 2018), essas sintomatologias irão ocorrer de mulher para mulher e serão experenciado conforme a história de vida de cada uma, levando em consideração as condições econômicas, culturais, sociais e fatores hereditários (Alcântara, Nascimento, \& Oliveira, 2020).

O objetivo desse estudo foi entender como as mulheres vivenciam o climatério, e conhecer as repercussões do climatério na vida das mulheres.

\section{Metodologia}

Trata-se de uma revisão integrativa da literatura que foi estruturada em seis fases distintas: identificação do tema e seleção da questão de pesquisa para a elaboração da revisão integrativa; estabelecimento de critérios para inclusão e exclusão de estudos/amostragem ou pesquisa de literatura; definição das informações a serem extraídas dos estudos 
selecionados/categorização dos estudos; avaliação dos estudos incluídos na revisão integrativa; interpretação dos resultados e, apresentação da revisão/síntese do conhecimento (Mendes, Silveira, \& Galvão, 2008).

Para responder a pergunta da pesquisa "Como as mulheres vivenciam o climatério", utilizou-se a estratégia PICo (Ppopulação/mulheres; I -interesse/vivencia; Co- contexto/climatério). A busca foi realizada na base de dados da Lilacs Literatura Latino Americana e do Caribe em Ciência em Saúde e Bdenf - Base de Dados em Enfermagem entre os meses de agosto e setembro de 2021.

$\mathrm{Na}$ busca, utilizou-se os descritores controlados e não controlados, os MEsh e operadores booleanos AND e $O R$, conforme String de busca: ((Mulheres) OR (Women) OR (Meninas) OR (Mulher)) AND ((vivencia)) AND ((climatério) OR (Climacteric) OR (Mudança de Vida)).

Inicialmente foram resgatados 77 estudos, após aplicação dos critérios de inclusão (estudos que respondessem à pergunta da pesquisa, disponíveis na integra, nos idiomas inglês, português e espanhol) e exclusão (artigos duplicados, teses, dissertações, manuais e artigos que abordassem o climatério em mulheres com patologia como doenças coronarianas, hipertensão e obesidade), restaram 58 artigos, sendo 51 na Lilacs e 39 na Bdenf. Após para leitura dos títulos e resumos dos estudos selecionadas, restaram 25 estudos que compuseram a amostra final.

A coleta de dados foi realizado com auxílio de um formulário com perguntas semiestruturadas elaborado pelos pesquisadores e subdividido em duas partes: Parte I - Perfil das produções (ano de publicação, tipo de estudo, local da pesquisa, abordagem metodológica, objetivos) e Parte II - Resultados em evidência (a vivencia e repercussões do climatério.

Os dados coletados foram organizados e categorizados conforme a o referencial teórico de Minayo (2012), no qual deve se seguir os seguintes critérios para ocorrer: leitura compreensiva do material selecionado, exploração do material, elaboração da síntese interpretativa. Na leitura compreensiva do material selecionado escolhem-se os documentos e o material é analisado montando uma estrutura que servirá de base para interpretação, descrevendo o material a partir da perspectiva dos atores e das informações coletadas; na exploração do material, são realizadas várias leituras a fim de codificar os dados e agregá-los em unidades, devendo-se ir além das falas e do material coletado, caminhando na direção do que está explícito para o implícito, viabilizando a organização do conteúdo afim de se achar categorias para melhor análise dos achados; e na elaboração da síntese interpretativa segue-se o princípio da decomposição do conjunto do material da pesquisa em unidades, e é onde se desvelam as categorias.

\section{Resultados}

Para a realização desta pesquisa foram revisados 25 artigos científicos abaixo na Tabela 1 serão descritos os artigos e manual realizados para enfim chegar aos resultados e consequentemente estabelecer a discussão. Dos 26 estudos selecionados, observou-se que a maioria das publicações foi realizada entre os anos de 2019-2020, tendo destaque a Revista Ciência e Saúde Coletiva como mostra a tabela a seguir. 
Tabela 1- caracterização dos artigos inclusos na revisão integrativa (título, ano, autor e revista).

\begin{tabular}{|c|c|c|c|}
\hline TÍTULO & ANO & AUTOR & REVISTA \\
\hline $\begin{array}{l}\text { Climatério e menopausa: percepção de } \\
\text { mulheres usuárias da estratégia saúde da } \\
\text { família. }\end{array}$ & 2016 & Aranha, J. Et Al. & Temas em Saúde \\
\hline $\begin{array}{l}\text { Práticas de cuidado realizadas por } \\
\text { enfermeiros às mulheres no climatério. }\end{array}$ & 2016 & Silva, C. Et Al. & Revista contexto \& saúde \\
\hline $\begin{array}{l}\text { Qualidade de vida de mulheres } \\
\text { climatéricas. }\end{array}$ & 2017 & Assunção, D.F.S. Et Al. & Rev Soc Bras Clin Med \\
\hline $\begin{array}{l}\text { Análise da qualidade de sono em mulheres } \\
\text { climatéricas ativas e sedentárias. }\end{array}$ & 2017 & Portela, L.P.R. Et Al. & Cons Saúde \\
\hline $\begin{array}{l}\text { Mulher e climatério: concepções de } \\
\text { usuárias de uma unidade básica de saúde. }\end{array}$ & 2017 & Souza, S. S. Et Al. & Reprodução \& Climatério \\
\hline $\begin{array}{l}\text { Influência do climatério em } \\
\text { relacionamento conjugais perspectiva de } \\
\text { gênero. }\end{array}$ & 2018 & Carvalho, L.C Et Al. & Rev Rene \\
\hline Percepção da mulher acerca do climatério. & 2018 & Piecha, V.H. Et Al. & Rev Fun Care Online \\
\hline $\begin{array}{c}\text { Avaliação do perfil lipídico e } \\
\text { socioeconômico em mulheres climatérica } \\
\text { da Zona Norte de Teresina. }\end{array}$ & 2018 & Silva, S.R.S. Et $A l$. & Revista Nursing \\
\hline $\begin{array}{c}\text { Fatores associados à autopercepção } \\
\text { negativa de saúde em mulheres } \\
\text { climatéricas. }\end{array}$ & 2018 & $\begin{array}{l}\text { Silva,V.H; Rocha, J.S.B; } \\
\text { Caldera A.P. }\end{array}$ & Rev Ciência e Saúde Coletiva \\
\hline $\begin{array}{l}\text { Avaliação do risco de queda e equilíbrio } \\
\text { em mulheres no climatério. }\end{array}$ & 2018 & $\begin{array}{l}\text { Xavier, P.F.P.; Trindade, } \\
\text { A.P.N.T. }\end{array}$ & Revista Kairós-Gerontologia \\
\hline $\begin{array}{l}\text { A vivência do climatério por mulheres } \\
\text { atendidas em uma unidade básica de saúde } \\
\text { no município de Gurupi- TO }\end{array}$ & 2019 & Alcântara, Et Al. & Revista Amazônia Science \&Health \\
\hline $\begin{array}{c}\text { Percepção das mulheres cadastradas em } \\
\text { uma estratégia saúde da família acerca do } \\
\text { climatério. }\end{array}$ & 2019 & Baleeiro, C. Et Al. & Revista Uningá \\
\hline $\begin{array}{l}\text { Perda da qualidade do sono e fatores } \\
\text { associados em mulheres climatéricas. }\end{array}$ & 2019 & Lima A.M. Et $A l$. & Rev Ciência Saúde Coletiva \\
\hline Promoção à saúde da mulher: & 2019 & Oliveira, Et $A l$. & Braz. J. of Develop. \\
\hline
\end{tabular}

O conhecimento como estratégia de enfrentar os desafios de conviver com o climatério.

o período climatério sob ótica da mulher.

Conhecimento das mulheres e dos homens referente ao climatério e menopausa

Assistência de enfermagem a mulher Climatérica: estratégias de inclusão na Rotina das unidades básicas de saúde.

A influência dos sintomas climatérios na saúde da mulher.
Silva, J. K. M.; Freire, M.B.B; Nascimento, E.G.C.D.

Silva, M. G ; Dias, M. S; Oliveira, M. P.

Alcântara, L.L; Nascimento, L.C; Oliveira, V.A.

Carneiro, M. Et Al.

Figueiredo Júnior, J.C. Et Al.
Almanaque multidisciplinar de pesquisa

Revista Saber Digital

Rev Enferm Foco

Revista Extensão 


$\begin{array}{cccc}\begin{array}{c}\text { Função sexual e qualidade de vida de } \\ \text { mulheres climatéricas. }\end{array} & 2020 & \text { Meira, L.F. Et Al. } & \text { Rev Fisioter Bras } \\ \begin{array}{c}\text { Conhecimento do climatério entre usuárias } \\ \text { da estratégia saúde da família. }\end{array} & 2020 & \text { Silva, R.C.S Et Al. } & \text { Revista de Ciências da Saúde Nova } \\ \text { Esperança }\end{array}$

Fonte: Autores (2021).

A análise dos dados acerca da vivência da mulher no climatério possibilita a construção de 3 categorias práticas: Categoria 1: Vivenciando os sinais e sintomas do climatério; Categoria 2 - As Repercussões do climatério na vida social da mulher e Categoria 3 - o as repercussões do climatério nos aspectos psicológicos da mulher.

\section{Discussão}

\section{Categoria 1: Vivenciando os sinais e sintomas do climatério}

Nota-se que os principais sinais e sintomas que se apresentam nesse período são: fogachos, perda de libido, irritabilidade e insônia. A análise permitiu observar que a intensidade desses sintomas geralmente são intensos, porém, depende de mulher para mulher, ou seja, a intensidade de todo quadro sintomatológico pode depender da qualidade de vida da mulher, do meio em que se encontra, do emocional e também do seu conhecimento sobre o climatério. A perda da libido influência diretamente na vida sexual da mulher, interfere nas relações conjugais tendo uma percepção negativa sobre esse período.

O envelhecimento representa um processo fisiológico que necessita de um cuidado especial (Xavier \& Trindade, 2018), o climatério sendo um fenômeno endócrino traz para a mulher mudanças físicas, hormonais e psicossociais simultaneamente (Figueiredo et al., 2020). Ademais, as repercussões hormonais do climatério proveniente do caimento da produção do estradiol, acarretam em alterações cardiovasculares, ósseo, alterações no humor e apetite, no qual leva a mulher a vivenciar o climatério de vários modos impactando sua vida em diversos meios (Lima et al., 2017).

Logo, nos últimos anos tem levantado interrogações com relação a sintomatologia climatérica e sua tendência em comprometer ou não a qualidade de vida da mulher (Assunção, Íris, Barreto, Gonçalves \& Dias, 2017). Assim, se faz necessário reconhecer os fatores que comprometem a qualidade de vida dessas mulheres, a fim de formular estratégias para uma vivência desse período com mais facilidade (Luz \& Frutuoso, 2021).

Figueiredo Junior et al. (2020), afirma que no climatério a presença de sinais e sintomas aborrecedores, no qual é denominado síndrome climatérica, ocorre por conta da diminuição gradual dos hormônios produzido pelos ovários. Conforme Piecha, Ebling, Peiszak, Silva e Silva (2018), muitas mulheres passam pelo período sem apresentar nenhuma queixa, como 
também as mulheres que apresentam sintomatologias, os sinais e sintomas variam de leves a intensos, podendo ser transitório ou não-transitório, tornando o climatério como um período de vivência singular de mulher para mulher.

Os principais sinais e sintomas do climatério podem se ramificar em dois grupos, o de manifestações transitórias, dentre estas as menstruais, neurogênicas, psicogênicas, urogenitais, metabolismo lipídico, metabolismo ósseo e ganho de peso, e o de alterações não transitórias (Oliveira et al., 2019).

As manifestações climatéricas mais comuns relacionadas a fase da pré-menopausa é a irregularidade do ciclo menstrual, alterações de humor e fogachos, já na fase pós-menopausa éa perda da libido, dispareunia, disúria, ressecamento vaginal, urgência urinária, alterações psicológicas (Marinho, Alcântara, Lima \& Teixeira, 2018). O autor explica que esta vivência é singular e varia de acordo com a história de vida de cada uma delas, das condições econômicas, sociais, culturais e fatores hereditários (Marinho et al., 2019).

Joventino, Silva, Trigueiro e Pereira (2020) explica que os sintomas que ocorrem mais cedo no climatério é a sudorese e o fogachos, atingindo $75 \%$ a $85 \%$ das mulheres, ocasionado modificações na qualidade de vida, além das ondas de calor que dependendo da sua potência e frequência podem influenciar no sono e nas atividades do dia-a-dia, podendo ser causa de depressão e irritabilidade. Assim, conforme Lima et al. (2017), esses eventos causam muitas vezes situações de estresse no qual aumenta a quantidade de cortisol no organismo, esse aumento pode interferir diretamente no humor e na qualidade do sono.

\section{Categoria 2 - As Repercussões do climatério na vida social e sexualidade da mulher}

A partir dos dados observa-se que o período do climatério interfere no meio social da mulher pois, por comprometer a autoestima, devido aos inúmeros desafios que surgem nesse período, os relacionamentos conjugais, profissionais e familiar são prejudicados, seja por conta da vulnerabilidade psicológica que ocorre, ou do estresse ocorrente desse período pela irritabilidade e mudanças de humor, sintomas comuns do climatério que ajuda nesse processo.

Dentre todas essas alterações metabólicas e fisiológicas, o aumento do peso corporal ocorre devido ao menor gasto energético, sedentarismo e a queda da atividade metabólica (Piecha et al., 2018). Assim, no período do climatério e após a menopausa, as mulheres progridem com um aumento do colesterol total, LDL-C, diminuição do HDL-C e triglicérides, eventos que não são favoráveis a saúde (Silva, Lima, Albuquerque \& Lima 2018), também a flutuação hormonal possibilita à redistribuição do tecido adiposo e à massa magra influenciando na autoestima da mulher (Piecha et al., 2018).

As mulheres no período do climatério cruzam-se com novas circunstâncias que é capaz de coincidir com modificações no seu status sexual (Vieira et al., 2018), na compreensão do climatério incluso da cultura na qual a mulher está introduzida, a saída dos filhos(as) da residência, a chegada da adolescência, adoecimento dos pais e também os questionamentos do envelhecimento do corpo, motivos que acabam intervindo a autoestima (Piecha et al., 2018).

A Sexualidade nesse momento de vida da mulher destaca-se diretamente na qualidade de vida (Meira et al., 2020). Mulheres com idades de 35 a 59 anos apresentam entre 25\% e 33\% disfunções sexuais, tendo potencial a alcançar a margem dos 75\% entre as mulheres de 60 a 65 anos de idade, as modificações que ocorrem no período do climatério não resultam na diminuição do prazer (Oliveira et al., 2019), uma queixa comum das climatéricas, pode ser atribuída pela atrofia vaginal, perda da rugosidade, redução da secreção vaginal, todos esses fatores podem causar danos conjugais a mulher contribuindo negativamente a vivência desse período (Joventino et al., 2020).

Em contrapartida, existem mulheres que em decorrência da diminuição na obrigação maternal, profissional, juntamente a fatores como a ausência de sintomas ou a diminuição destes por tratamento ou mudanças melhorando a qualidade de vida, se dá uma chance para viver uma fase afeto/sexual de forma positiva (Silva et al., 2020). 
Um estudo realizado por Oliveira et al., (2019) aponta que os sintomas do climatério podem afetar tanto na sexualidade como no padrão de desempenho sexual da mulher, referenciando uma diminuição do padrão de desempenho/satisfação sexual da mulher climatérica, de acordo com o avanço da idade. Assim, Maciel (2021) e Oliveira et al., (2019) concordam quanto ao desejo sexual durante o climatério sofre influências, pois diversos fatores psicossociais e biológicos não seguem um padrão, na qual essa questão afeta na relação da mulher com o companheiro, consequentemente em suas experiências anteriores.

Logo, pode-se afirmar que a sexualidade no período do climatério ressalta na vida da mulher tanto biologicamente com o déficit hormonal, como social (Baleeiro et al., 2019), ou seja, evidencia-se que nesse período a sexualidade da mulher climatérica é vivenciada de uma forma, onde há vários tabus e preconceitos na qual se tem a perspectiva que no período do climatério a mulher torna-se assexuada, que o sexo é prazeroso apenas na fase jovem (Baleeiro et al., 2019).

\section{Categoria 3 - o as repercussões do climatério nos aspectos psicológicos da mulher}

Ao que foi analisado e levantado pela pesquisa, o climatério interfere de forma negativa no que diz respeito ao contexto psicológico da mulher, o processo de envelhecimento junto as várias concepções físicas e fisiológicas alteradas por conta desse período, a falta de conhecimento e compreensão por parte da família dificulta o enfrentamento dessa fase. Logo, todo esse cenário leva a mulher a ter baixa autoestima e até desenvolver depressão e ansiedade em casos intensos, fatores que faz com que as mulheres sofram ainda mais nesse período.

Silva, Dias e Oliveira (2019) enfatizam que em geral a mulher tem uma visão negativa deste período do seu ciclo vital em virtude dos sentimentos de medo e insegurança comuns nessa fase da vida. Diante disso, Soares et al. (2018), destaca a importância do apoio familiar entra como peça-chave ao apoio a mulher, o cuidado a climatérica ousa ir além do cuidado físico, o contexto familiar também se torna fundamental para essa assistência, o cuidar da mulher nesse momento parte também da compreensão familiar, pois, eles fazem parte do cotidiano e convívio da mulher.

O que mais amedronta as mulheres ao relatar que estão nesse período, não é apenas os sintomas ou as enfermidades que ali são conectadas a fase do climatério e envelhecimento, Carneiro et al., (2020) atribui esse medo a existência de algo que se torna desconhecido a mulher, esse ponto demonstra que os fatores psicológicos influenciam muito na vida da mulher, e é seguindo esse contexto que o apoio positivo da família ajuda a mulher enfrentar com mais facilidade esses obstáculos.

Um estudo realizado por Silva, Oliveira, Souza, Silva e Ykeda (2020) em uma unidade de saúde de João Pessoa-PB, evidencia que a maioria das mulheres climatéricas não possuem clareza quando se trata das modificações hormonais acerca do climatério, a maior parte dessas mulheres desconhecem qualquer possibilidade de tratamento para amenizar os sintomas. Maciel et al. (2021), diz que a vivência acerca do climatério é dessemelhante de mulher para mulher, há mulheres que aceitam o período, ou por não ter interferência em sua vida ou por apenas aceitar ser uma fase fisiológica, outras vê em o climatério como algo que muda totalmente suas vidas, enxergando essa fase de forma negativa.

Para Aranha, Lima, Lima e Nobre, (2018) sintomas oriundos da menopausa e do climatério são capazes de modificar a qualidade das relações pactuadas no trabalho e na família, interferir na condição de satisfação das mulheres e abalar seu cotidiano habitual e/ou relações interpessoais. Maciel et al. (2021) afirma que vivenciar essa fase do climatério sem preparação pode ocorrer dificuldade para encarar os desafios que surgem e, ainda, comprometer a autoestima, os relacionamentos e a qualidade de vida. Há poucas informações a respeito desse acontecimento, no Brasil, abalam mais as famílias de baixa renda Maciel et al. (2021). Além disto Silva, Rocha e Caldeira (2018) afirmam que, a baixa ou nenhuma escolaridade e a ausência de procura por serviços de saúde favorece para falta de incompreensão da menopausa. 
Em contrapartida, de acordo com o estudo realizado por Carvalho et al. (2018) em uma unidade de saúde de Teresina, Piauí, Brasil, evidencia que algumas das mulheres climatéricas recebe do seu companheiro carinho, ajuda, compreensão, respeito, além de apoio para um melhor convívio com a família diante dos seus momentos de alterações sintomatológicas, ademais Carvalho et al. (2018) diz também que outras mulheres evidencia o momento sexual, de forma reduzida, pois conforme irão envelhecendo, vai diminuindo o interesse pelo ato sexual por conta de alterações dessa fase, e mesmo diante de todos essas dificuldades, os parceiros entendem seu momento.

Meira et al. (2020) afirma que alterações físicas, psicológicas e hormonais presentes no período climatérico podem suceder em importantes impacto para sexualidade feminina e convívio com a companheira e enfatiza que o apoio do conjugue em ações voltadas ao climatério favorece positivamente na vivencia dessa fase pelo parceiro. Contudo, Carvalho et al. (2018) explica a influencia desse período no relacionamento do casal, e inevitável, no entanto singular, pois depende da maneira como cada conjugues vivenciam o próprio relacionamento e como em qualquer outra fase de modificações e reajustamento deve ocorre face as transformações.

Logo, outras mulheres vivencia a atividade sexual, de maneira menos frequente, pois para algumas, com a diminuição ou a ausência da libido, evidenciado que em alguns relações sexuais ocorre de maneira passiva, de forma que acontece apenas a satisfação do cônjuge, interferindo de forma direta na vivencia do relacionamento marido- mulher (Maciel et al., 2021).

Para Piecha et al. (2018) cada mulher experiência essa fase com sinais e sintomas diferentes, conforme seu grau de entendimento sobre a referente etapa e da sua preparação diante das transformações que sugiram pois a veemência com que elas ocorrem está referidas com as condições culturais, psicológicos e sociais, ou seja, Piecha et al. (2018) afirma que as mulheres com maior despreparo e negação sobre a referente fase, as sintomatologias poderão acontecer em proporções maiores do que para as mulheres que estão mais preparadas para o climatério.

A ausência de informações sobre climatério em seus vários aspectos impacta diretamente no seu autocuidado (Alcântara, Nascimento, \& Oliveira, 2020), assim, se torna importante a educação em saúde (Silva et al., 2016). A medida em que a menopausa se aproxima, surgem dúvidas, o conhecimento sobre todo esse processo tende a diminuir a ansiedade que se gera na mulher (Silva, Frere \& Nascimento, 2019). A qualidade de vida sendo um fator essencial para a diminuição dos sintomas do período e da ansiedade consequente do climatério, como atividade física, diminuem consideravelmente de intensidade (Portela et al., 2017). Apesar de não ser um evento patológico, esse período é vivenciado de forma patológica pelas mulheres (Souza et al., 2017).

\section{Considerações Finais}

O estudo aponta que a grande maioria das mulheres vivenciam o climatério de forma negativa, pois elas sofrem influenciam de fatores, seja emocional, social, cultural, psicológico, como também algumas manifestações sintomatologias que ocorre como a diminuição do desejo sexual ou ausência da libido, envelhecimento do corpo, estresse, fogachos e baixo autoestima, fatores esses que lhe trazem sofrimento e acabam proporcionando o distanciamento do conjugue.

Foi evidenciado que cada mulher experencia o climatério de maneira singular, assim como as sintomatologias que têm um impacto diferente na vida de cada uma e que nem todas tem o conhecimento necessário para experenciar essa fase de maneira mais positiva. É de grande importância o apoio da família e do companheiro nessa etapa, pois são vistos como peças essenciais nesta fase, dando apoio emocional para a mulher, de certo modo sendo compreensivo a ponto de entender que esta etapa da vida é complexa. 
Assim, os profissionais atuantes que acompanham a climatérica, devem obter conhecimentos mais aprofundados sobre o tema, entender que a mulher nesse período pode vivenciar de várias formas e com isso ter experiências singulares e, portanto, cada singularidade deve-se ter uma intervenção humanizada e qualificada, para assim melhorar o atendimento e a vivência da mesma nesse período.

\section{Referências}

Alcântara, L. L., Nascimento, L. C, \& Oliveira, V. A. (2020). Conhecimento das mulheres e dos homens referente ao climatério e menopausa. Rev Enferm Foco, 11(1), 44-49.

Aranha, J. D. S, Lima, C. B. D, Lima, M. N. F. D. A, \& Nobre, J. D. O. C. (2016). Climatério e menopausa: Percepção de mulheres usuárias da estratégia saúde da família. Temas em Saúde, 16(2), 588-612.

Assunção, D. F. D. S., Pires, D. H. K., Barreto, E. D. L. Gonçaves, F. D. A., \& Dias, R. D. S. (2017). Qualidade de vida de mulheres climatéricas. Rev Soc Bras Clin Med, 15(2), 80-83

Baleeiro, C. G. B., Gomes, D. D. M., Alves, C, D, R., Barbosa, G. P., Souza, M. S., \& Teles, M. A. B. (2019). Percepção das mulheres cadastradas em uma estratégia saúde da família acerca do climatério. Revista Uningá, 56(S2), 100-106.

Carneiro, M. D. E. S. G., Silva, P. A., Markus, G. W. S., Pereira, R. A., Couto, G. B. F. D., \& Dias, A. K. (2020). Assistência de enfermagem a mulher Climatérica: estratégias de inclusão na Rotina das unidades básicas de saúde. Revista Extensão. Guaraí-TO, 4(2).

Carvalho, M. L. D., Jr., Parente, A. D. C. M., \& Sales, J. C. e S. (2018). Influência do climatério em relacionamento conjugais perspectiva de gênero. Rev Rene, 19, e. 32617. doi:10.15253/2175-6783.20181932617

Da Silva, M. G., Dias, M. S., \& Oliveira, M. P. (2019). O período climatério sob ótica da mulher. Revista Saber Digital, 12(1), 29-38. https://revistas.faa.edu.br/index.php/SaberDigital/article/view/723

Jro., Morais, F. D.V., Ribeiro, W. A., Pereira, G. L. F. D. L., Felicio, F. D. C., Andrade, D. L. B. (2020). A influência dos sintomas climatérios na saúde da mulher. Revista Nursing, 23(264), 4002-4007.

Joventino, M. L. D. S., Silva, R. C. S. D., Trigueiro, D. R. S., \& Pereira, V. C. L. D. S. (2020). Conhecimento do climatério entre usuárias da estratégia saúde da família. Revista de Ciências da Saúde Nova Esperança, 18(3), 166-175. doi:10.17695/rcsnevol18n3p166-175

Lima, A. M., Rocha, J. S. B., Reis, V. M. C. P., Silveira, M. F., Caldeira, A. P., Freitas, R. F., \& Popoff, D, A, V. (2019). Perda da qualidade do sono e fatores associados em mulheres climatéricas. Rev Ciência Saúde Coletiva, 24(7), 2667-2678. doi: org/10.1590/1413-81232018247.19522017

Luz, M. M. F., \& Frutuoso, M. F. P. (2021). O olhar do profissional da atenção primária sobre o cuidado à mulher climatérica. Interface, 25(Supl.). doi: https://doi.org/10.1590/interface.200644

Maciel, J. B. J., Sipaúba, A. J. C., Andrade, T. L. D. C., Barroso, H. L. M. R., Amorim, J. F. D., Silva, K. D. S. M., \& Souza, A. D. S. (2021). Vivência e concepção da mulher acerca do climatério: Uma revisão bibliográfica. Research, Society and Development, 10(6), e. 9710615557. doi: http://dx.doi.org/10.33448/rsd-v10i6.15557

Marinho, H. S. G., Alcântara. D. S., Lemos. R. B. D., \& Texeira. A. C. D. S. (2018). A vivência do climatério por mulheres atendidas em uma unidade básica de saúde no município de Gurupi- TO. Revista Amazônia Science \& Health, 6(1), 43-47. doi:10.18606/2318-1419

Meira, L.F., Morais. K. C. S. D., Sousa. N. A. D.; \& Ferreira. J. B. (2021). Função sexual e qualidade de vida de mulheres climatéricas. Rev Fisioter Bras, 20(1), 101-108. doi: http://dx.doi.org/10.33233/fb.v20i1.2672

Mendes, K.D.S., Silveira, R.C.C.P., \& Galvão, C.M. (2008). Revisão integrativa: método de pequisa para a incorporação de evidências na saúde e na enfermagem. Revista texto e contexto, 17(4). doi: https://doi.org/10.1590/S0104-07072008000400018

Minayo, M. C. D. S. (2012). Análise qualitativa: Teoria, passos e fidedignidade. Ciência \& saúde coletiva, 17(3), 621-626.

Oliveira, A. R., Melo. A. P. M. D., Lopes, K. R., Anjos, L. S., Silveira, M. D. M., Valério, P. G. P., . . Viana, P. C. G. (2019). Promoção à saúde da mulher: Desmistificando o climatério. Braz. J. of Develop, 5(10), 21431-21442. doi:10.34117/bjdv5n10-297

Piecha, V. H., Ebling, S. B. D., Pieszak, G. M., Silva, M. M. D., \& Silva, S. D. O. (2018). Percepção da mulher acerca do climatério. Revista de Pesquisa: Cuidado é Fundamental Online, 10(4), 906-912. doi: 10.9789/2175-5361.2018.v10i4.906-912

Portela, L. P. R., Silva, 1. D. C., Rodrigues, T. F., Oliveira, L. M. N. D., \& Fº (2017). Análise da qualidade de sono em mulheres climatéricas ativas e sedentárias. Cons Saúde, 16(2), 274-280.

Silva, T. C. D., Bisognin, P., Prates, L. A., Possati, A., \& Ressel, L. B. (2016). Práticas de cuidado realizadas por enfermeiros às mulheres no climatério. Revista contexto \& saúde, 16(30), 21-27.

Silva, C. D. S., Oliveira, B. C. D., Souza, S. M. D. O., Silva, H. G. N., \& Ykeda, D. S. C.S. (2020). Estudo comparativo da qualidade do sono e insônia entre mulheres no climatério e com cilo menstrual regular. Rev Pesqui Fisioter, 10(2) 163-171. doi: 10.17267/2238-2704rpf.v10i2.2779 
Research, Society and Development, v. 10, n. 13, e184101321093, 2021

(CC BY 4.0) | ISSN 2525-3409 | DOI: http://dx.doi.org/10.33448/rsd-v10i13.21093

Silva, J. K. M., Freire, M. B. B., \& Nascimento, E. G. C. D. (2019). O conhecimento como estratégia de enfrentar os desafios de conviver com o climatério. Almanaque multidisciplinar de pesquisa, 6(1).

Silva, S. R. S., Lima, H. M. R., Albuquerque, J. O. L., \& Lima, A. B. D. M. (2018). Avaliação do perfil lipídico e socioeconômico em mulheres climatérica da Zona Norte de Teresina. Revista Nursing, 21(241), 2201-2210.

Silva, V. H., Rocha, J. S. B., \& Caldera, A. P. (2018). Fatores asociados à autopercepção negativa de saúde em mulheres climatéricas. Rev Ciência e Saúde Coletiva, 23(5), 1611-1620. doi: 10.1590/1413-81232018235.17112016

Souza, S. S. D., Santos, R. L. D., Santos, A. D. F. D., Barbosa, M. D. O., Lemos, I. C. S., \& Machado, M. D. F. A. S. (2017). Mulher e climatério: Concepções de usuárias de uma unidade básica desaúde. Reprodução \& Climatério, 32(2), 85-89.

Vieira, T. M. M., Araujo, C. R. D., Souza, E. C. D. S. D., Costa, M. A. R., Teston, E. F., Benedetti, G. M. D. S., \& Marquete, V. F. (2018). Vivenciando o climatério: percepções e vivência de mulheres atendidas na atenção básica. Enferm. Foco, 9(2), 40-45.

Xavier, P. F. D. P., Trindade, A. P. N. T. D. (2018). Avaliação do risco de queda e equilíbrio em mulheres no climatério. Revista Kairós-Gerontologia, 21(2), 155-170. doi: http://dx.doi.org/10.23925/2176-901X.2018v21i2p155-170 